\title{
NUTRIENTES FOLIARES DE ESPÉCIES ARBÓREAS NA MATA ATLÂNTICA: EFEITO DO TAMANHO DO FRAGMENTO
}

\author{
LEAF NUTRIENTS IN ATLANTIC FOREST TREE SPECIES: FRAGMENT SIZE EFFECT
}

\author{
Ana Paula da Silva ${ }^{1}$ Dora Maria Villela ${ }^{2}$
}

\begin{abstract}
RESUMO
O presente estudo foi desenvolvido em fragmentos de Mata Atlântica na região de baixadas do Rio de Janeiro, inseridos na área de proteção da bacia do Rio São João. O objetivo deste estudo foi testar a hipótese de que o tamanho do fragmento altera a concentração de macronutrientes em folhas de duas das principais espécies arbóreas (Guarea guidonea e Cupania oblongifolia) ocorrentes nessa área. Folhas adultas de Guarea guidonea e Cupania oblongifolia foram coletadas em três fragmentos pequenos (23 - 33 ha), três médios (130 - 155 ha) e três grandes (500 - 2300 ha). Carbono, N, Ka, Ca e, Mg foram determinados. O tamanho do fragmento em geral não afetou o estado nutricional nas folhas das espécies estudadas. No entanto, a maior concentração de $\mathrm{Mg}$ em folhas de Cupania oblongifolia nos fragmentos menores $(5,0 \pm 0,8$ $\mathrm{mg} / \mathrm{g})$ em relação aos fragmentos médios $(4,0 \pm 0,3 \mathrm{mg} / \mathrm{g})$ e grandes $(3,5 \pm 0,7 \mathrm{mg} / \mathrm{g})$ indica um efeito do tamanho do fragmento sobre este elemento.
\end{abstract}

Palavras-chave: ciclagem de nutrientes; Cupania oblongifolia; Floresta Tropical; fragmentação florestal.

\begin{abstract}
The study was made in Atlantic forest fragments in the lowland region of the protected area of São João river basin, Rio de Janeiro state, Brazil. The aim of the study was to test the hypothesis that fragment size alters macro-nutrients concentrations in leaves of the main tree species (Guarea guidonea e Cupania oblongifolia) in the area. Adult leaves of Guarea e Cupania were sampled in three small (23- 33 ha), three medium (130 - $155 \mathrm{ha})$ and three large (500 - $2300 \mathrm{ha})$ fragments. Carbon, $\mathrm{N}, \mathrm{Ca}, \mathrm{Mg}$ and $\mathrm{K}$ were determined. The results showed that, in general, the reduction of the fragment size did not affect the nutritional status of the species studied. Nevertheless, the higher Mg concentrations in Cupania oblongifolia leaves in the small fragments $(5.0 \pm 0.8 \mathrm{mg} / \mathrm{g})$ compared with the medium $(4.0 \pm 0.3 \mathrm{mg} / \mathrm{g})$ and large $(3.5 \pm 0.7 \mathrm{mg} / \mathrm{g})$ ones indicated an effect of the reduction of the fragment area over this element.
\end{abstract}

Keywords: Cupania oblongifolia; forest fragmentation; Guarea guidonea; leaf nutrients.

\section{INTRODUÇÃO}

A limitação de nutrientes tem sido demonstrada amplamente na literatura como um fator importante para a produtividade de ecossistemas florestais (PROCTOR, 1989; VITOUSEK, 2004), especialmente em solos tropicais. Isto se deve à baixa disponibilidade de nutrientes encontrada nesses solos, onde a vegetação se desenvolve dentro de um possível quadro de limitação nutricional (VITOUSEK, 2004). Esta pode ser avaliada através da disponibilidade de nutrientes no solo associada à concentração de nutrientes em folhas (AERTS e CHAPIM, 2000; VITOUSEK, 2004). Entretanto, plantas que crescem em solos pobres em nutrientes podem apresentar maior concentração de nutrientes em relação a plantas de solos com alta disponibilidade (AERTS e CHAPIM, 2000),

1 Bióloga, Dra., Professora do Instituto Federal de Educação, Ciência e Tecnologia do Rio de Janeiro (IFRJ), Campus Nilópolis, 1045, CEP 26530-060, Nilópolis (RJ), Brasil. ana.paula@ifrj.edu.br

2 Bióloga, Dr ${ }^{\mathrm{a}}$., Professora Associada do Laboratórios de Ciências Ambientais, Universidade Estadual do Norte Fluminense Darcy Ribeiro, 2000, CEP 28013-600, Campos dos Goytacazes (RJ), Brasil. dora@uenf.br 
em função do desenvolvimento de estratégias de conservação de nutrientes nos tecidos foliares (CARRERA et al., 2003). Espécies de estádio sucessional secundário podem apresentar maior eficiência de uso de nutrientes em relação às espécies de estádio primário (KELLMAN, 1969). Dessa forma, o padrão de ciclagem de nutrientes pode ser influenciado pela estrutura e composição florística da comunidade vegetal, na qual as espécies dominantes podem ter um papel muito importante (VILLELA e PROCTOR, 2002).

Os processos de fragmentação e o consequente efeito de borda têm causado a redução da biodiversidade e da biomassa de florestas tropicais (KAPOS, 1997; CARVALHO et al., 2006; RODRIGUES e NASCIMENTO, 2006), o que pode interferir na dinâmica do ecossistema (MARTINELLI et al., 2000). Estudos demonstram que fragmentos menores estão mais sujeitos aos efeitos da fragmentação (CAMARGO e KAPOS, 1995; RODRIGUES e NASCIMENTO, 2006), tais como mudanças no padrão da vegetação (LAURANCE et al., 1998; PROCÓPIO-OLIVEIRA et al., 2008) e na dinâmica do ecossistema (MARTINELLI et al., 2000). Essas mudanças podem alterar o padrão da ciclagem de nutrientes (VITOUSEK, 2004) e consequentemente levar a uma limitação de nutrientes ao crescimento vegetal, podendo afetar a regeneração da comunidade vegetal e a composição e riqueza de espécies da área.

Procópio-Oliveira et al. (2008), estudando remanescentes florestais na região de baixadas do Rio de Janeiro, verificaram alterações na estrutura da vegetação e na dinâmica da floresta decorrentes dos efeitos da fragmentação. O estoque de serapilheira em tais fragmentos também foi influenciado, sendo este menor nos fragmentos pequenos do que nos fragmentos grandes, devido ao pior estado de conservação dos fragmentos pequenos. Apesar do avanço e interesse em estudos relacionados ao efeito da fragmentação em florestas, poucos visaram compreender este impacto sobre o estoque e a ciclagem de nutrientes, tais como os relacionados a estudos de decomposição na Amazônia (DIDHAM, 1998; RUBINSTEIN e VASCONCELOS, 2005) e produção de serapilheira na Amazônia (VASCONCELOS e LUIZÃO, 2004) e na Mata Atlântica (NASCIMENTO, 2005; PORTELA e SANTOS, 2007; VIDAL et al., 2007).

Alguns estudos avaliaram a concentração de nutrientes foliares em floresta tropicais
(DRECHSEL e ZECH, 1991; BOEGER et al., 2005; ESPIG, et al., 2008; BAMBI, et al., 2011). Entretanto, não se conhece o efeito da fragmentação florestal sobre a concentração de nutrientes em folhas. Visando preencher parte desta lacuna no conhecimento, o presente estudo objetivou avaliar a concentração de macronutrientes em folhas verdes de duas espécies arbóreas (Guarea guidonea (L.) Sleum. e Cupanea oblongifolia Mart.) em fragmentos de Mata Atlântica que se diferenciam quanto à classe de tamanho, na região de baixadas do Rio de Janeiro, e testar a hipótese de que as concentrações de nutrientes nas folhas das duas espécies estudadas são alteradas pelo tamanho dos fragmentos.

\section{MATERIAIS E MÉTODOS}

O presente estudo foi realizado na região de baixadas de Imbaú, município de Silva Jardim - RJ, inseridos na Área de Proteção Ambiental da bacia do rio São João. Esta região apresenta vários remanescentes florestais de Mata Atlântica de baixada a submontana, com vegetação caracterizada como Floresta Ombrófila Densa. Estes remanescentes se encontram em diferentes estádios sucessionais de regeneração natural (CARVALHO et al., 2006; 2007; 2008; PROCÓPIO-OLIVEIRA et al., 2008). O clima da região é caracterizado como tropical úmido, apresentando uma estação seca no inverno (PRIMO e VOLKER, 2003). A precipitação oscila entre 1500 e $2000 \mathrm{~mm}$, sendo os meses de junho a agosto os menos chuvosos e de menores temperaturas, e os meses de novembro a março os mais chuvosos e de maiores temperaturas (PRIMO e VOLKER, 2003).

Para o presente trabalho foram utilizados nove fragmentos desta região classificados em classes de tamanho: três pequenos ("Afetiva" 25,5 ha, "Estreito" 23,3 ha, "Vendaval" 33,5 ha); três médios ("Andorinhas" 145 ha, "Imbaú" 130 ha, "Sítio Professor" 155 ha); e três grandes ("Rio Vermelho" 406 ha, "União 500" ha e "Rebio" 2300 ha). Esses fragmentos são circundados por matrizes de pasto e capoeira e culturas de subsistência (CARVALHO et al., 2006) e distam de 2 a $61 \mathrm{~km}$ entre si, apresentando diferenças quanto ao grau de isolamento (PROCÓPIO-OLIVEIRA et al., 2008). O fragmento de Mata Atlântica da Rebio (2300 ha), localizado no distrito de Rocha Leão, município de Rio das Ostras - RJ, é considerado entre os demais fragmentos o mais preservado (RODRIGUES e 
NASCIMENTO, 2006). O fragmento Sítio do Professor é uma Reserva Particular do Patrimônio Natural (RPPN). Os demais fragmentos estão em áreas de fazendas particulares, portanto, ainda sujeitas às pressões antrópicas Procópio-Oliveira et al. (2008).

Os nove fragmentos estudados são importantes áreas de ocorrência do mico-leãodourado onde foram desenvolvidos outros estudos com objetivo de avaliar a qualidade do habitat desta espécie tais como a avaliação da estrutura e diversidade florística dos fragmentos (CARVALHO et al., 2006; 2007; 2008) e avaliação da biomassa arbórea e estoques de nutrientes (LIMA et al., 2011; PROCÓPIO-OLIVEIRA et al., 2008) e biomassa radicular fina (LIMA et al., 2011).

Os solos dos fragmentos estudados são do tipo Latossolo Amarelo Distrófico e Latossolo Vermelho Amarelo Distrófico, com exceção do fragmento Estreito (EST) que apresenta solo hidromórfico (Gleissolo Háplico) de acordo com Lima (2008). Os teores de macronutrientes encontrados nesses solos, em geral, são semelhantes entre as classes de fragmentos, $\mathrm{K}\left(0,1-0,2 \mathrm{cmol}_{\mathrm{c}}\right.$. $\left.\mathrm{kg}^{-1}\right), \mathrm{C}(2,0-3,1 \%), \mathrm{N}(0,2-0,3 \%)$ com exceção do $\mathrm{Mg}$ que foi significativamente maior nos fragmentos médios $\left(5,3\right.$ cmolc. $\left.\mathrm{kg}^{-1}\right)$ em relação aos fragmentos pequenos $\left(3,9 \mathrm{cmol} \cdot \mathrm{kg}^{-1}\right)$ e grandes $(3,1$ $\left.\mathrm{cmol} \cdot \mathrm{kg}^{-1}\right)$ e do Ca que foi significativamente maior nos fragmentos pequenos $\left(0,5 \mathrm{cmol} \cdot \mathrm{kg}^{-1}\right)$ que nos médios $\left(0,4 \mathrm{cmol}_{\mathrm{c}} \cdot \mathrm{kg}^{-1}\right)$ e nos grandes $\left(0,3 \mathrm{cmol}_{\mathrm{c}} \cdot \mathrm{kg}^{-}\right.$ ${ }^{1}$ ) (PROCÓPIO-OLIVEIRA et al., 2008).

Os resultados de estrutura e florística obtidos por Procópio-Oliveira et al. (2008) nos mesmos fragmentos do presente estudo demonstraram que as espécies mais importantes foram: Guarea guidonea; Cupania oblongifolia; Plathymenia foliolosa; Guapira nitida; Siparuna guianensis. Nestes também foi encontrada menor diversidade florística e área basal em comparação com a mata madura da Rebio (2300 ha). As matas destes fragmentos se encontram em estágio sucessional secundário, com uma elevada densidade de espécies secundárias iniciais, resultante da fragmentação. No entanto, apesar do tamanho pequeno e do mau estado de conservação, esses fragmentos apresentam características importantes para preservação (PROCÓPIO-OLIVEIRA et al., 2008).

Para o presente estudo foram coletadas aleatoriamente vinte folhas adultas (caracterizadas pela coloração verde-escura e totalmente expandidas), na porção inferior de cada uma das copas a partir do quarto nó, voltadas para a face norte, de cada uma das seis árvores de Guarea guidonea e seis de Cupania oblongifolia, por fragmento. As amostragens foram realizadas no interior (100 $\mathrm{m}$ da borda) de cada um dos nove fragmentos estudados, em 25 de agosto de 2005. A escolha das espécies baseou-se no alto valor de cobertura (Guarea guidonea $=22,41 \%$; Cupania oblongifolia $=11,12 \%$ ) e de dominância relativa (Guarea guidonea $=14,55 \%$; Cupania oblongifolia $=5,04 \%$ ) apresentado por essas espécies em todos os fragmentos, exceto na Rebio (2300 ha), além do fato de ambas serem caracterizadas como sucessionais secundárias (CARVALHO et al., 2006). As folhas de Cupania oblongifolia não foram coletadas no fragmento "União 500", pois neste não foram encontrados indivíduos desta espécie.

No laboratório, as folhas foram lavadas com água corrente e água deionizada, secas em estufa a $60^{\circ} \mathrm{C}$ até atingir peso constante, moídas em moinho elétrico de faca e homogeneizadas em grau e pistilo (ALLEN, 1989). As determinações de $\mathrm{C}$ e $\mathrm{N}$ totais foram feitas em amostras secas utilizando-se um analisador de elementos CHNS/O (Perkin Elmer 2400). Para a extração de $\mathrm{Ca}, \mathrm{Mg}$ e $K$ subamostras $(0,20 \mathrm{~g})$ foram digeridas em solução de ácido sulfúrico concentrado $(350 \mathrm{ml})$, água oxigenada 100 volumes $(420 \mathrm{ml})$, selênio $(0,42 \mathrm{~g})$ como catalisador e sulfato de lítio $(14 \mathrm{~g})$ (ALLEN, 1989). As determinações foram feitas em espectrofotometria de emissão por plasma induzido (ICP/AES-Varian). Todo o procedimento analítico foi realizado no Laboratório de Ciências Ambiental (LCA) na UENF, RJ. Os coeficientes de variação entre as réplicas analíticas foram: $\mathrm{N} 2-8 \%, \mathrm{~K} 6,6-$ $14 \%$; Ca $1,4-10,5 \%$; Mg 3,4-14\% e C $1-4 \%$.

Para a normatização dos dados, estes passaram por transformação arco-seno. As comparações das concentrações de nutrientes entre as classes de fragmentos foram feitas através de análises de variância agrupada (ANOVA nested design), considerando cada fragmento como fator randômico dentro de cada categoria de tamanho (fator fixo). A comparação entre espécies dentro de cada categoria de tamanho foi feita através do teste $t$ de Student (ZAR, 1999). Foram utilizadas correlações de Spearman para avaliar a relação da concentração de nutrientes nas folhas das espécies com os tamanhos dos fragmentos e com as variáveis dos solos de cada fragmento, obtidos por ProcópioOliveira et al. (2008). 


\section{RESULTADOS E DISCUSSÃO}

As concentrações de nutrientes nas folhas de Guarea guidonea e Cupania oblongifolia, em geral não diferiram significativamente entre as classes de tamanho dos fragmentos (Tabela 1), com exceção do $\mathrm{Mg}$, não evidenciando um efeito do tamanho do fragmento sobre os status nutricional destas espécies.

Não houve uma correlação significativa entre a concentração de $\mathrm{Ca}$ em folhas de Cupania oblongifolia $\left(\mathrm{r}^{2} 0,053, \mathrm{p}=0,582\right)$ ou de Guarea guidonea $\left(\mathrm{r}^{2} 0,094, \mathrm{p}=0,422\right)$ com o tamanho do fragmento. Entretanto, verificou-se uma correlação significativamente positiva entre a concentração de $\mathrm{Ca}$ em folhas de Cupania oblongifolia e a concentração deste no solo dos fragmentos $\left(\mathrm{r}^{2} 0,993\right.$, $\mathrm{p}=0,049$ ), indicando que a maior concentração de $\mathrm{Ca}$ nas folhas desta espécie nos fragmentos pequenos é em decorrência da maior disponibilidade deste elemento no solo dos fragmentos pequenos em relação aos fragmentos médios e grandes (PROCÓPIO-OLIVEIRA et al., 2008). Já em folhas de Guarea guidonea, não foi observada correlação com a concentração de $\mathrm{Ca}$ no solo $\left(r^{2} 0,507, p=0,495\right)$.
As folhas de Cupania oblongifolia apresentaram concentrações de $\mathrm{Mg}$ significativamente maiores nos fragmentos pequenos em relação aos fragmentos médios e grandes, os quais apresentaram concentrações similares entre si. Já as folhas de Guarea guidonea apresentaram concentração de $\mathrm{Mg}$ semelhantes entre as classes de tamanho (Tabela 1). Porém, não foi observada uma correlação significativa entre as concentrações de $\mathrm{Mg}$ em folhas de Cupania oblongifolia com o tamanho do fragmento $\left(\mathrm{r}^{2} 0,058\right.$, $\mathrm{p}=0,564)$. Ao contrário de $\mathrm{Ca}$, as concentrações de Mg nas folhas de Cupania oblongifolia não responderam à diferença na disponibilidade deste no solo, já que estes valores não se correlacionaram significativamente com a concentração de $\mathrm{Mg}$ nos solos $\left(\mathrm{r}^{2} 0,031, \mathrm{p}=0,887\right)$.

A maior concentração de $\mathrm{Mg}$ em folhas de Cupania oblongifolia nos fragmentos pequenos se assemelha ao padrão encontrado por Nascimento (2005) na borda do fragmento da Rebio União, onde houve uma maior concentração deste elemento em folhas da serapilheira em relação ao interior, o que segundo a autora, pode estar relacionado à maior quantidade de espécies pioneiras encontradas na borda por Rodrigues e Nascimento (2006).

TABELA 1: Concentração de C e N (\%) e Ca, $\mathrm{Mg}, \mathrm{K}(\mathrm{mg} / \mathrm{g})$ e razão $\mathrm{C} / \mathrm{N}$ em folhas verdes de Guarea guidonea e Cupania oblongifolia em três classes de tamanho de fragmentos de Mata Atlântica de baixada na região de Imbaú, Silva Jardim - RJ.

TABLE 1: Concentration of the $\mathrm{C}$ and $\mathrm{N}(\%)$ e $\mathrm{Ca}, \mathrm{Mg}, \mathrm{K}(\mathrm{mg} / \mathrm{g})$ and $\mathrm{C} / \mathrm{N}$ in green leaves of the Guarea guidonea and Cupania oblongifolia in three Atlantic forests fragments in the lowland region of the Imbaú, Silva Jardim, RJ state, Brazil.

\begin{tabular}{|c|c|c|c|c|c|c|}
\hline $\begin{array}{l}\text { Classe de tamanho } \\
\text { dos fragmentos }\end{array}$ & $\mathrm{N}$ & K & $\mathrm{Ca}$ & $\mathrm{Mg}$ & $\mathrm{C}$ & $\mathrm{C} / \mathrm{N}$ \\
\hline & $---\%---$ & & $\mathrm{cmol}_{\mathrm{c}} \cdot \mathrm{dcm}^{3}$ & & $-\ldots \%-\ldots$ & - \\
\hline \multicolumn{7}{|l|}{ Pequenos } \\
\hline Guarea guidonea & $4,6 \pm 0,2 \mathrm{~A} 1$ & $22,8 \pm 6,2 \mathrm{~A} 1$ & $6,1 \pm 2,0 \mathrm{~A} 1$ & $3,0 \pm 0,1 \mathrm{~A} 1$ & $44,4 \pm 2,5 \mathrm{~A} 1$ & $10 \mathrm{~A} 1$ \\
\hline Cupania oblongifolia & $4,3 \pm 0,2 \mathrm{~b} 1$ & $31,4 \pm 17,6$ b1 & $7,9 \pm 1,2 \mathrm{a} 1$ & $5,0 \pm 0,8 \mathrm{a} 2$ & $43,3 \pm 2,8 \mathrm{~b} 1$ & $10 \mathrm{~b} 1$ \\
\hline \multicolumn{7}{|l|}{ Médios } \\
\hline Guarea guidonea & $5,2 \pm 0,1 \mathrm{~A} 1$ & $29,2 \pm 7,6 \mathrm{~A} 1$ & $7,0 \pm 0,9 \mathrm{~A} 1$ & $2,9 \pm 0,6 \mathrm{~A} 1$ & $45,8 \pm 0,7 \mathrm{~A} 1$ & $9 \mathrm{~A} 1$ \\
\hline Cupania oblongifolia & $4,7 \pm 0,2 \mathrm{~b} 2$ & $37,3 \pm 11,3 \mathrm{~b} 1$ & $6,3 \pm 2,0$ a1 & $4,0 \pm 0,3 \mathrm{~b} 2$ & $45,5 \pm 1,2 \mathrm{~b} 1$ & $10 \mathrm{~b} 1$ \\
\hline \multicolumn{7}{|l|}{ Grandes } \\
\hline Guarea guidonea & $5,0 \pm 1,1 \mathrm{~A} 1$ & $20,4 \pm 10,6 \mathrm{~A} 1$ & $5,2 \pm 1,2 \mathrm{~A} 1$ & $3,0 \pm 0,3 \mathrm{~A} 1$ & $49,5 \pm 7,5 \mathrm{~A} 1$ & $10 \mathrm{~A} 1$ \\
\hline Cupania oblongifolia & $4,4 \pm 0,2 \mathrm{~b} 2$ & $23,8 \pm 7,9$ b1 & $5,0 \pm 1,6$ al & $3,5 \pm 0,7 \mathrm{~b} 1$ & $45,9 \pm 0,2 \mathrm{~b} 1$ & $10 \mathrm{~b} 1$ \\
\hline
\end{tabular}

Em que: Letras diferentes na mesma coluna indicam diferenças significativas $(p<0,05)$ entre as classes de tamanho dos fragmentos (ANOVA nested design, $\mathrm{n}=3$ ), para cada uma das espécies. Números diferentes na mesma coluna indicam diferenças significativas $(\mathrm{p}<0,05)$ entre espécies em cada classe de tamanho dos fragmentos (Teste $t, n=3$ ). 
Tomando-se como pressuposto que os fragmentos pequenos do presente estudo podem ser considerados uma borda em toda sua extensão, no que se refere aos parâmetros estruturais (CARVALHO et al., 2006; PROCÓPIO-OLIVEIRA et al., 2008), tal processo pode explicar a maior concentração de $\mathrm{Mg}$ em folhas de Cupania oblongifolia nos fragmentos menores, como consequência da possibilidade de maior entrada de radiação nestas áreas mais abertas associada a maior taxa fotossintética (LARCHER, 2000). Estudos de Boeger et al. (2005) nos quais avaliaram os nutrientes foliares de espécies arbóreas em Floresta Ombrófila Densa em três estádios sucessionais no sul do Brasil, encontrou uma maior concentração de $\mathrm{Mg}$ em folhas das espécies do estádio inicial, seguido do intermediário.

Ambas as espécies do presente estudo, apresentaram concentrações de C, N e K semelhantes entre as classes de tamanho dos fragmentos (Tabela 1). As concentrações de $\mathrm{N}$ nas folhas também não se correlacionaram positivamente com o tamanho dos fragmentos (Guarea guidonea: $\mathrm{r}^{2} 0,007, \mathrm{p}=$ 0,828; Cupania oblongifolia: $\left(\mathrm{r}^{2} 0,031, \mathrm{p}=0,673\right)$, nem com a concentração de N total no solo (Guarea guidonea: $\mathrm{r}^{2} 0,599, \mathrm{p}=0,436$; Cupania oblongifolia: $\left(r^{2} 0,440, p=0,537\right)$. Da mesma forma, não houve correlação significativa entre as concentrações foliares de C (Cupania oblongifolia $=r^{2} 0,100, p$ $=0,445 ;$ Guarea guidonea $\left.=\mathrm{r}^{2} 0,021, \mathrm{p}=0,704\right)$ e de $\mathrm{K}\left(\mathrm{r}^{2} 0,181, \mathrm{p}=0,292, \mathrm{p}=0,445 ; \mathrm{r}^{2} 0,118\right.$, $\mathrm{p}$ $=0,363$ ) com o tamanho do fragmento, ou com a concentração desses elementos no solo (C, Cupania oblongifolia $=\mathrm{r}^{2} 0,105, \mathrm{p}=0,789$, Guarea guidonea $=\mathrm{r}^{2} 0,676, \mathrm{p}=0,385 ; \mathrm{K}$, Cupania oblongifolia $=$ $\mathrm{r}^{2} 0,968, \mathrm{p}=0,113$, Guarea guidonea $=\mathrm{r}^{2} 0,763$, $\mathrm{p}=0,323)$.

Os resultados das concentrações destes nutrientes no solo dos fragmentos pequenos também não apresentaram diferenças quando comparado aos fragmentos grandes (PROCÓPIO-OLIVEIRA et al., 2008; LIMA et al., 2011). No entanto, em estudo de produção de serapilheira realizado nos mesmos fragmentos (SILVA, 2009), foi verificado um menor aporte de nutrientes nos fragmentos pequenos em relação ao fragmento da Rebio (2.300 ha), sendo este reflexo da menor produção de serapilheira total nos fragmentos pequenos (7,2 a 8,2 t/ha) (SILVA, 2009) em relação ao fragmento grande da Rebio (9,38 t/ha) (NASCIMENTO, 2005). O menor aporte de nutrientes nos fragmentos pequenos indicou um efeito negativo do tamanho sobre a entrada de nutrientes através da serapilheira nos fragmentos estudados (SILVA, 2009). Outros estudos também têm verificado alterações em aspectos importantes da ciclagem de nutrientes causadas por impactos antrópicos (VILLELA et al., 2006). Os que buscam avaliar o efeito do tamanho do fragmento sobre a produção de serapilheira, têm encontrado uma menor produção em fragmentos menores em relação aos fragmentos maiores (VIDAL et al., 2007; PORTELA e SANTOS 2007; SILVA 2009), o que pode favorecer um maior aporte de nutrientes nesses fragmentos maiores e resultar em uma maior disponibilidade de nutrientes no solo de fragmentos grandes. Nascimento (2005) encontrou uma menor produção de folhas da serapilheira e um maior aporte de $\mathrm{Mg}$ e $\mathrm{Na}$ na borda do que no interior do fragmento grande da Rebio, atribuído ao efeito de borda neste.

Comparando-se as espécies entre si, em cada classe de tamanho de fragmento, verificou-se que as folhas de Cupania oblongifolia apresentaram maiores concentrações de $\mathrm{Mg}$ em relação às de Guarea guidonea nos fragmentos pequenos e médios (Tabela 1). Isto indica umamaior exigência da espécie por este nutriente, o que está provavelmente relacionada à característica de Cupania oblongifolia como espécie de sub-bosque Carvalho et al. (2006). Este resultado demonstra a capacidade diferenciada das espécies florestais em concentrar nutrientes, conforme observado por Cunha et al. (2009) ao analisarem a concentração de nutrientes em folhas de espécies arbóreas em fragmentos florestais de Mata Atlântica, no Parque Estadual do Desengano, município de Santa Maria Madalena, região norte-fluminense, RJ, Brasil.

A concentração de $\mathrm{N}$ foi significativamente maior em folhas de Guarea guidonea em relação à Cupania oblongifolia nos fragmentos médios e grandes, o que pode refletir a eficiência de retranslocação de $\mathrm{N}$ verificada para esta espécie (36 a 48\%) (SILVA, 2009). Portanto, o teor de N em folhas de Guarea guidonea parece ser mais influenciado pela eficiência de retranslocação do que pela disponibilidade deste elemento no solo (SILVA, 2009). Dessa forma, espera-se que possíveis limitações de $\mathrm{N}$ no solo não sejam refletidas na concentração destes elementos nas folhas de Guarea guidonea (AERTS e CHAPIN, 2000; BAMBI et al., 2011). Os resultados das concentrações N no solo dos fragmentos pequenos também não indicam uma limitação deste elemento quando comparado aos fragmentos grandes (PROCÓPIO-OLIVEIRA et al., 2008; LIMA et al., 2007). 
A alta concentração de $\mathrm{N}$ encontrada nas folhas das espécies estudadas quando comparada com outras florestas tropicais, evidencia o potencial de ciclagem interna deste elemento pelas plantas (SILVA, 2009). YUAN et al. (2005), avaliando a retranslocação de $\mathrm{N}$ em floresta seca do semiárido da China, verificaram que folhas com altas concentrações de $\mathrm{N}$ foram as que apresentaram mecanismo eficiente de retranslocação.

As concentrações de $\mathrm{Ca}$ e $\mathrm{Mg}$ encontradas nas folhas das espécies deste estudo, estão na faixa de outras áreas de florestas tropicais (Tabela 2). As concentrações de $\mathrm{Mg}$ nos fragmentos estudados se assemelham aos valores reportados por Boeger et al. (2005) para floresta de Mata Atlântica em estádio sucessional inicial e intermediário, e acima dos valores encontrados por Thompson et al. (1992) para floresta amazônica sempre-verde, caracterizada como madura (Tabela 2). Os valores de $\mathrm{K}$ encontrados neste estudo estão acima da maioria dos reportados para outras florestas tropicais (Tabela 2), assemelhando-se apenas à floresta Amazônica, indicando que o $\mathrm{K}$ não é limitante nos fragmentos estudados.

A razão $\mathrm{C} / \mathrm{N}$ nas folhas das espécies estudadas não apresentou diferença estatística entre

TABELA 2: Concentrações médias (mg/g) de macronutrientes em folhas de formações florestais tropicais. TABLE 2: Macronutrient concentrations ( $\mathrm{mg} / \mathrm{g}$ ) in leaves of tropical rain forests.

\begin{tabular}{|c|c|c|c|c|c|c|c|c|c|}
\hline \multirow{2}{*}{ Tipo de Floresta } & \multirow{2}{*}{ Local/Tipo } & \multirow{2}{*}{$\begin{array}{l}\mathrm{N} \cdot \mathrm{de} \\
\text { espécies }\end{array}$} & $\mathrm{Ca}$ & $\mathrm{Mg}$ & $\mathrm{K}$ & $\mathrm{C}$ & $\mathrm{N}$ & $\mathrm{C} / \mathrm{N}$ & \multirow{2}{*}{ Autor } \\
\hline & & & & -- & $---m$ & /g - - & --- & & \\
\hline \multirow{7}{*}{ Ombrófila Densa } & Silva Jardim - RJ & & & & & & & & \\
\hline & Fragmentos Pequenos & 2 & 7,0 & 4,0 & 27,1 & 438,0 & 44,6 & 10 & Este Estudo \\
\hline & Fragmentos Médios & 2 & 6,6 & 3,4 & 33,2 & 457,0 & 50,6 & 9 & Este Estudo \\
\hline & Fragmentos Grandes & 2 & 5,3 & 3,2 & 21,0 & 479,0 & 46,6 & 9 & Este Estudo \\
\hline & $\begin{array}{c}\text { Paraná } \\
\text { Estádio inicial }\end{array}$ & 8 & 8,5 & 4,5 & 3,2 & 522,0 & 11,8 & 48 & $\begin{array}{l}\text { Boeger et al. } \\
\quad(2004)\end{array}$ \\
\hline & Estádio intermediário & 8 & 6,1 & 3,5 & 4,3 & 510,0 & 12,0 & 44 & \\
\hline & Estádio avançado & 8 & 7,4 & 2,8 & 5,2 & 506,0 & 14,0 & 38 & \\
\hline $\begin{array}{l}\text { Floresta de } \\
\text { Transição } \\
\text { (Terra firme) }\end{array}$ & Norte do Mato Grosso & 3 & 115,8 & - & 6,6 & 328,3 & 17,0 & 19 & $\begin{array}{l}\text { Bambi et al. } \\
\text { (2011) }\end{array}$ \\
\hline \multirow{4}{*}{ Ombrófila Densa } & Pernambuco & & & & & & & & $\begin{array}{l}\text { Espig et al. } \\
\quad(2008)\end{array}$ \\
\hline & Ilha do Cardoso - SP & 10 & 16,62 & 3,65 & 12,23 & - & 21,23 & - & $\begin{array}{c}\text { Moraes e } \\
\text { Domingos } \\
(1997)\end{array}$ \\
\hline & Mata de Restinga & 10 & 10,3 & 4,9 & 9,8 & & 31,7 & & \\
\hline & Mata Atlântica & 10 & 6,6 & 4,1 & 3,7 & & 12,9 & & \\
\hline $\begin{array}{l}\text { Ombrófila Densa } \\
\text { de Terras Baixas }\end{array}$ & Roraima & 77 & 4,7 & 2,4 & 10,3 & & 18,8 & & $\begin{array}{c}\text { Thompson et al. } \\
\text { (1992) }\end{array}$ \\
\hline Restinga Paludosa & Paraná & 9 & 4,9 & 2,9 & 5,2 & - & - & - & $\begin{array}{c}\text { Britez et al. } \\
\text { (1997) }\end{array}$ \\
\hline Terra firme & Amazônia & 1 & 7,7 & 3,3 & 7,1 & - & 19,0 & - & $\begin{array}{l}\text { Martinelli et al. } \\
(2000)\end{array}$ \\
\hline $\begin{array}{l}\text { Tropical Úmida } \\
\text { secundária }\end{array}$ & Amazônia & 1 & 14,6 & 5,7 & 20,9 & - & - & - & $\begin{array}{c}\text { Herrera e } \\
\text { Finegan (1997) }\end{array}$ \\
\hline
\end{tabular}


os fragmentos. No entanto, esses valores encontramse bem abaixo de valores encontrados por Boeger et al. (2005) para áreas de Mata Atlântica (Tabela 2). Essa comparação evidencia a alta qualidade das folhas das espécies estudadas no que diz respeito à razão $\mathrm{C} / \mathrm{N}$, o que consequentemente favorece processos de decomposição e liberação de $\mathrm{N}$, pois resíduos vegetais com altos valores da razão $\mathrm{C} / \mathrm{N}$, resultantes de baixas reservas de nitrogênio, favorecendo a imobilização (VITOUSEK, 2004). A taxa de decomposição de folhas de Guarea guidonea nos fragmentos pequenos e grandes foi alta em relação a valores encontrados para outras áreas de Mata Atlântica (SILVA, 2009), podendo ser resultado da qualidade superior das folhas desta espécie.

O fato de uma análise geral ter demonstrado uma baixa influência da fragmentação sobre o estado nutricional foliar de Guarea guidonea e Cupania oblongifolia nos fragmentos estudados, sugere que é preciso considerar que os efeitos da fragmentação podem ter variado diferentemente ao longo do espaço e do tempo nos fragmentos estudados, dificultando a simples associação com o tamanho do fragmento. Fraga (2007) não observou relações entre o tamanho do fragmento e a densidade, riqueza e diversidade de plântulas de espécies arbóreas nos mesmos fragmentos do presente estudo, o que, segundo a autora, parecem estar mais relacionados ao histórico de preservação destes. Portanto, outros fatores, além do tamanho do fragmento, podem estar envolvidos no processo de fragmentação da Mata Atlântica em questão, dificultando a simples relação com o tamanho do fragmento em si e os processos relacionados à ciclagem de nutrientes.

\section{CONCLUSÕES}

As concentrações dos nutrientes nas folhas de Guarea guidonea e Cupania oblongifolia, estudadas em fragmentos de Mata Atlântica Ombrófila com diferentes tamanhos, na Bacia do rio São João, município de Silva Jardim - RJ em geral não foram relacionadas com o tamanho dos fragmentos. Apenas a concentração de $\mathrm{Mg}$ em folhas de Cupania oblongifolia foi superior nos fragmentos pequenos (33,5 ha), indicando que o $\mathrm{Mg}$ nesta espécie parece responder às variações relacionadas ao tamanho dos fragmentos estudados.

As folhas de Cupania oblongifolia, de uma forma geral, apresentaram maiores concentrações de $\mathrm{Mg}$ em relação às de Guarea guidonea, enquanto as de Guarea guidonea foram maiores em concentração de N. As folhas das espécies estudadas apresentaram altas concentrações de $\mathrm{N}$ e K quando comparadas com outras áreas de florestas tropicais e menor $\mathrm{C} / \mathrm{N}$, o que indica uma boa qualidade nutricional dessas espécies nos fragmentos de Mata Atlântica estudados.

\section{AGRADECIMENTOS}

Os autores agradecem ao laboratório de Ciências ambientais da UENF pelo apoio logístico; a Pós-graduação em Ecologia e Recursos Naturais (PPGRN) e a UENF pela bolsa de doutorado fornecida à primeira autora; ao Instituto Chico Mendes (Rebio-União) pelo apoio logístico; a Associação Mico-Leão-Dourado, CEPF, FNMA, FAPERJ, CNPq pelo auxílio financeiro ao projeto; aos técnicos Helmo Carvalho, José Wanderley e Gerson do Laboratório de Ciências Ambientais da UENF pelo apoio no trabalho de campo; aos técnicos de laboratório Arizoli Gobo, Cristiano Peixoto Maciel, Marcelo Almeida e Ana Paula Pedrosa, pelo apoio recebido nas análises químicas.

\section{REFERÊNCIAS BIBLIOGRÁFICAS}

AERTS, R.; CHAPIN, F. S. The mineral nutrition of wild plants revisited: re-evaluation of processes and patterns. Pp. 1-67. In: FITTER AH, RAFFAELLI DG(Ed). Advances in Ecological Research. San Diego, Academic Press, 2000.

ALLEN, S, E. Chemical Analysis of Ecological Materials. 2nd. ed. Blackwell Scientific Publications. London, 1989.

BAMBI, P. et al. Decomposição e redistribuição de nutrientes das folhas de espécies da floresta de transição Amazônia - Cerrado, MT. Ciência e Natura, Santa Maria, v. 33, n. 1, p. 17 - 31, 2011. BOEGER, M. R. T; WISNIEWSKI, C.; REISSMANN, C. B. Nutrientes foliares de espécies arbóreas de três estádios sucessionais de floresta ombrófila densa no sul do Brasil. Acta. Botânica Brasilica., v. 19, n. 1, p. 167-181, 2005.

BRITEZ, R. M. et al. Chemical characterization of two forests on the coastal plains of the Ilha do Mel, Paraná, Brazil. Developments in Plants and Soil Sciences, v.78, p. 461-462, 1997.

CAMARGO, J. L. C.; KAPOS, V. Complex edge effects in soil moisture and microclimat in Central Amazonian forest. Journal of Tropical Ecology, v. 11, p. 205-221, 1995. 
CARVALHO, F. A.; NASCIMENTO, M. T.; BRAGA, J. M. A. Composição e riqueza florística do componente arbóreo da Floresta Atlântica submontana na região de Imbaú, município de Silva Jardim, RJ. Acta Botânica Brasílica, v. 20, n. 3, p. 741-754, 2006.

CARVALHO, F. A.; NASCIMENTO, M. T.; BRAGA, J. M. A. Estrutura e composição florística do estrato arbóreo de um remanescente de Mata Atlântica. Revista Árvore, Viçosa, v. 31, n. 4, p.717-730. 2007.

CARVALHO, F. A.; NASCIMENTO, M. T.; OLIVEIRA-FILHO, A. T. Composição, riqueza e heterogeneidade da flora arbórea da bacia do rio São João, RJ, Brasil. Acta Botânica Brasil. v. 22, n. 4, p. 929-940. 2008.

CARRERA, A. L. et al. (2003) Relationship between plant nitrogen conservation strategies and the dynamics of soil nitrogen in the arid Patagonian Monte, Argentina. Plant Soil, v. 255, p. 595-604.

CUNHA, G. M. et al. Biomassa e estoque de carbono e nutrientes em florestas montanas da mata atlântica na região norte do estado do Rio de Janeiro. Revista Brasileira de Ciência do Solo, v. 33, p. 1175-1185, 2009.

DIDHAM, R. K. Altered leaf-litter decomposition rates in Tropical forest fragments. Oecologia, v. 116, p. 397-406, 1998.

DRECHSEL, P.; ZECH, W. Foliar nutrient levels of broadleaved tropical trees: a tabular review. Plant and Soil, v. 131, p. 29-46, 1991.

ESPIG, S. A. et al. Composição e eficiência da utilização biológica de nutrientes em fragmento de Mata Atlântica em Pernambuco. Ciêncial Florestal, Santa Maria. v. 18, n. 3, p. 309-316, 2008.

FRAGA, M. M. Regeneração natural de espécies arbóreas em fragmentos de Mata Atlântica na APA da bacia do rio São João, RJ. 93 f. Dissertação (Mestrado). Universidade Estadual do Norte Fluminense, Campos dos Goytacazes, RJ, 2007.

HERRERA, B.; FINEGAN, B. Substrate conditions, foliar nutrients and the distributions of two canopy tree species in a Costa Rican secondary rain forest. Plant and Soil., v. 191, p. 259-267, 1997.

KAPOS, V. et al. Edge-related changes in environment and plant responses due to forest fragmentation in central Amazonia. In: LAURANCE, W. F. \& BIERREGAARD-JR, R. O. (Ed). Tropical Forest Remnants: ecology, management, and conservation of fragmented communities. Chicago University Press. Chicago., 1997. p. 33-44.
KELLMAN, M.C. Some environmental components of shifting cultivation in upland Mindanao. Journal of Tropical Geography., v. 28, p. 40-56, 1969.

LARCHER, W. Ecofisiologia Vegetal. São Carlos, Rima. 2000. 531 p.

LAURANCE, W. F. et al. Rain forest fragmentation and the dynamics of Amazonian tree communities. Ecology., p. 2032-2040, 1998.

LIMA, J. A. S. et al. Biomassa radicular fina em fragmentos da Mata Atlântica Fluminense. Floresta (UFPR. Impresso). , v. 41, p. 27 - 38, 2011. LIMA, J. A. S.; VILLELA, D. M., MANZATTO, C.V. Biomassa arbórea em fragmentos da mata Atlântica fluminense. Boletim de Pesquisa e Desenvolvimento (Embrapa Solos. Online)., v. 127, p. 1 - 24, 2008.

MARTINELLI LA. et al. Variation in Nutrient distribution and Potential Nutrient Losses by Selective Logging in a Humid Tropical Forest of Rondônia, Brazil. Biotropica., v. 32, n. $4^{\text {a }}$, p. 597-613, 2000.

MORAES, R. M.; DOMINGOS, M. Elementos minerais em folhas de espécies arbóreas de Mata Atlântica e Mata de Restinga, na Ilha do Cardoso, SP. Revista Brasileira de Botânica., v. 20, n. 2, p. 133-138, 1997.

NASCIMENTO, A. C. P. Produção e aporte de nutrients da serrapilheira produzida em um fragmento de Mata Atlântica da Reserva Biológica União, RJ. Dissertação (Mestrado em Biociências e Biotecnologia). UENF, Campos dos Goytacazes, RJ, 2005.

PORTELA, R. D. E. C. Q.; SANTOS, F. A. M. Produção e espessura da serapilheira na borda e interior de fragmentos florestais de mata atlântica de diferentes tamanhos. Revista Brasileira de Botânica., v. 30, n. 2, p. 271-280, 2007.

PRIMO, P. B.; VOLKER, C. M. Bacias hidrográficas dos rios São João e das Ostras: águas, terras e conservação ambiental. Rio de Janeiro, Consórcio Intermunicipal Lagos São João, 2003.

PROCÓPIO-DE-OLIVEIRA, P. et al. Qualidade do habitat na área de ocorrência do mico-leão-dourado. In: PROCÓPIO-DE-OLIVEIRA P., GRATIVOL A. D., MIRANDA C. R. (Ed). Conservação do micoleão-dourado., 2008. p. 14-39.

PROCTOR, J. Mineral nutrients in tropical forest and savana ecosystems. Blackwell Scientific Publications. Oxford London Edinburgh. 1989. p. 473 p.

RODRIGUES P. J. F. P.; NASCIMENTO M. T. 
Fragmentação florestal: Breves Considerações teóricas sobre efeito de borda. Rodriguésia., v. 57, n. 1, p. 63-74, 2006.

RUBINSTEIN, A.; VASCONCELOS, H. L. Leaflitter decomposition in Amazonian forest fragments.

Journal of Tropical Ecology., v. 21, p. 699-702, 2005.

SILVA, A. P. Ciclagem de nutrientes na Mata Atlântica de baixada na APA da bacia do rio São João, RJ: efeito do tamanho do fragmento. Tese (Doutorado). UENF,Campos dos Goytacazes, RJ.

THOMPSON, J. etal. Ecological studies on a lowland evergreen rain forest on Maracá Island,Roraima, Brazil. I. Physical environment, forest structure and leaf chemistry. Journal of Ecology., v. 80, p. 689-703, 1992.

VASCONCELOS, H. L.; LUIZÃO F. J. Litter production and litter-nutrient concentrations in fragmented Amazonian Landscape: Edge and soil effects. Ecologycal Applications., v, 14, n. 3, p. 884-892, 2004.
VIDAL, M. M. et al. Produção de serapilheira em floresta Atlântica secundária numa paisagem fragmentada (Ibiúna, SP): importância da borda e tamanho dos fragmentos. Revista Brasileira de Botânica., v. 30, n. 3, p. 521-532, 2007.

VILLELA, D. M.; PROCTOR, J. Leaf decomposition and monodominant in the Peltogyne Forest of Maracá Island, Brazil. Biotropica., v. 34, p. 334-347, 2002.

VILLELA, D. M. et al. Effect of selective logging on forest structure and nutrient cycling in a seasonally dry brazilian Atlantic forest. Journal of Biogeography., v. 33, p. 506-616, 2006.

VITOUSEK, P. M. Nutrient cycling and limitation: Hawai'I as a model system. Princeton: Environmental Institute series. 2004. 223 p.

YUAN, Z. L. I. L. et al. Soil characteristics and nitrogen resorption in Stipa krylovii native to northern China. Plant and Soil., v. 273, p. 257-268, 2005.

ZAR, J. H. Biostatistical analysis. NewJersey: Prentice Hall International, 1984. 718. p. 